\title{
Extensive Pulmonary Laceration in Pediatric Trauma
}

\author{
Patricia DePuy ${ }^{b}$ Daniel W. Young ${ }^{d}$ Steven M. Rowe ${ }^{a-c}$

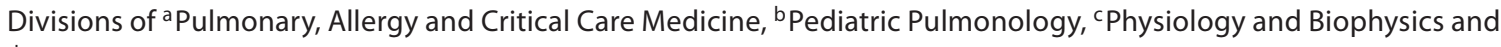 \\ dPediatric Radiology, University of Alabama at Birmingham, Birmingham, Ala., USA
}

A 5-year-old male with a history of frequent upper respiratory infections presented following a motor vehicle crash as an unrestrained, ejected passenger. CT of the chest and abdomen showed a small liver laceration, multifocal peripheral lung opacities (fig. 1, black arrows) and extensive lung laceration (fig. 1, white arrow). The patient required supplemental oxygen, but no surgical intervention. Due to fever, the patient underwent bronchoscopy revealing hemorrhagic large airways, and bronchoalveolar lavage showed erythrocytes and few inflammatory cells; all stains and cultures were negative for microorganisms. After $72 \mathrm{~h}$, an air-fluid level worsened within the posterior lung cyst. Following treatment with antibiotics, repeat imaging showed complete resolution of lung contusions and improvement of the laceration. This case provides an example of pulmonary contusion, the most common injury reported in severe pediatric blunt trauma, accompanied with extensive pulmonary laceration, an infrequent manifestation of chest trauma that can mimic lung abscess. Pulmonary laceration often occurs in coup/contra-coup areas, is frequently most severe in the posterior lung (due to shear stress) and can be complicated by hemorrhage or superinfection. As opposed to adults, pulmonary laceration occurs in children due to shear effects propagated by a relatively compliant chest wall. As in our case, most pulmonary lacerations will improve with appropriate supportive care.

Supported by NIH K23 DK075788-01 (S.M.R.).
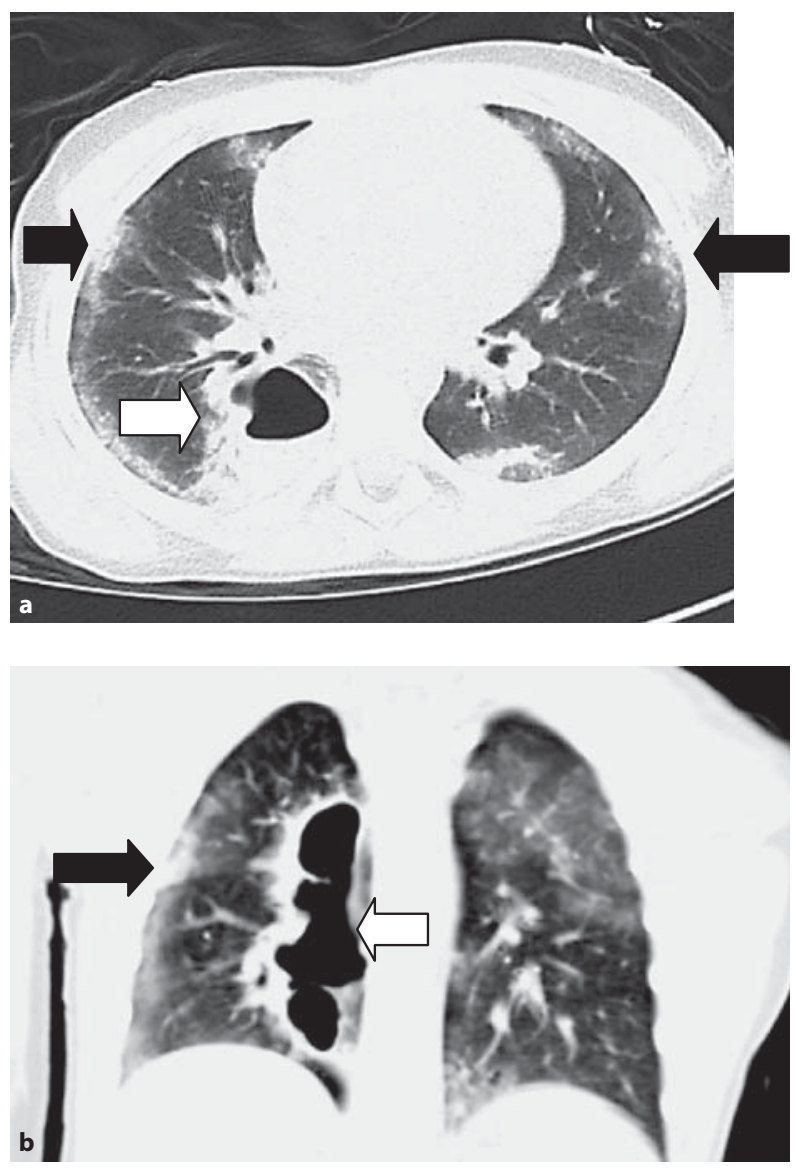

Fig. 1. Axial (a) and coronal (b) thoracic CT images demonstrating multifocal pulmonary contusions (black arrows) and a large posterior lung laceration (white arrows).

\section{References}

KARGER

๑) 2008 S. Karger AG, Basel

Fax +41613061234 E-Mail karger@karger.ch www.karger.com www.karger.com/res
Assist. Prof. Steven M. Rowe, MD, MSPH

768 MCLM

1918 University Boulevard

Birmingham, AL 35233 (USA)

Tel. +1 205934 9640, Fax +1 205934 7593, E-Mail smrowe@uab.edu 